\title{
GTPase activity-coupled treadmilling of the bacterial tubulin FtsZ organizes septal cell wall synthesis
}

\author{
Xinxing Yang ${ }^{1}$, Zhixin Lyu ${ }^{1,{ }^{*},}$ Amanda Miguel ${ }^{2,{ }^{*}, \text { Ryan McQuillen }}{ }^{1}$, Kerwyn Casey \\ Huang $^{2,3, \dagger}$, and Jie Xiao ${ }^{1, \dagger}$ \\ ${ }^{1}$ Department of Biophysics and Biophysical Chemistry, Johns Hopkins University School of \\ Medicine, Baltimore, MD 21205, USA \\ ${ }^{2}$ Department of Bioengineering, Stanford University, Stanford, CA 94305, USA \\ ${ }^{3}$ Department of Microbiology and Immunology, Stanford University School of Medicine, Stanford, \\ CA 94305, USA
}

\begin{abstract}
The bacterial tubulin FtsZ is the central component of the cell division machinery, coordinating an ensemble of proteins involved in septal cell wall synthesis to ensure successful constriction. How cells achieve this coordination is unknown. We found that in Escherichia coli cells, FtsZ exhibits dynamic treadmilling predominantly determined by its guanosine triphosphatase activity. The treadmilling dynamics direct the processive movement of the septal cell wall synthesis machinery but do not limit the rate of septal synthesis. In FtsZ mutants with severely reduced treadmilling, the spatial distribution of septal synthesis and the molecular composition and ultrastructure of the septal cell wall were substantially altered. Thus, FtsZ treadmilling provides a mechanism for achieving uniform septal cell wall synthesis to enable correct polar morphology.
\end{abstract}

The tubulin homolog FtsZ (1) is the central component of the cell division machinery in nearly all walled bacterial species (2). During division, FtsZ polymerizes on the cytoplasmic face of the inner membrane to form a ring-like structure, the Z-ring (3), and recruits more than 30 proteins to the division site (4). Many of these proteins are involved in septal synthesis of the peptidoglycan (PG) cell wall (4). The guanosine triphosphatase (GTPase) activity of FtsZ is highly conserved $(5,6)$, and the binding and hydrolysis of GTP underlie the dynamic assembly and disassembly of FtsZ $(7,8)$. Although several proposed mechanisms revolve around GTPase activity-dependent constriction force generation by the Z-ring (9), in E. coli the GTPase activity of FtsZ appears nonessential for cell division and

\footnotetext{
${ }^{\dagger}$ Corresponding author. kchuang@ @stanford.edu (K.C.H.); xiao@jhmi.edu (J.X.).

*These authors contributed equally to this work.

SUPPLEMENTARY MATERIALS

www.sciencemag.org/content/355/6326/744/suppl/DC1

Materials and Methods

Figs. S1 to S17

Tables S1 to S6

References (37-50)

Movies S1 to S19
} 
does not dictate the cell constriction rate $(10,11)$. Thus, the biological function of FtsZ's GTPase activity in bacterial cell division remains elusive.

To understand the role of GTPase activity in division, we characterized Z-ring dynamics in live E. coli BW25113 cells by using total internal reflection fluorescence (TIRF) microscopy to monitor the fluorescence of an FtsZ-GFP fusion protein (fig. S1) (12). FtsZ-GFP was expressed in the presence of endogenous, unlabeled wild-type FtsZ at $46 \pm 4.3 \%$ of total cellular FtsZ concentration (mean $\pm \mathrm{SD}, n=3$; fig. S2). The integrated TIRF intensity of the Z-ring exhibited large, approximately periodic fluctuations (Fig. 1, A and B; fig. S3; and movies S1 and S2). We observed similar behaviors with other fluorescent fusions (figs. S4 and S5 and movie S3) $(13,14)$ but not with fixed cells (fig. S4G) or fluorescent beads (fig. $\mathrm{S} 4 \mathrm{H})$, suggesting periodic assembly and disassembly cycles of FtsZ polymers in the Z-ring.

The power spectral densities (PSDs) of individual cells revealed clear peaks in the intensity fluctuations (Fig. 1C). The mean PSD curve of all cells showed a lognormal-like distribution (Fig. 1D, green curve) with a peak period of $115 \pm 10 \mathrm{~s}$ (mean \pm SEM; table S1) after subtracting the contribution of Z-ring dynamics due to stochastic subunit exchange (Fig. 1D, red curve) $(7,8,12)$. We calibrated the FtsZ-GFP intensity with cellular expression levels of FtsZ and FtsZ-GFP (figs. S1 and S6) (12), to estimate that $683 \pm 439$ FtsZ molecules (mean \pm SD; Fig. 1E) assembled in each TIRF intensity peak (Fig. 1B); these likely correspond to groups of smaller FtsZ clusters previously described in superresolution imaging studies (1517). The assembly and disassembly rates of these FtsZ polymers (Fig. 1B) were essentially the same (Fig. 1F), indicating a dynamic steady state of polymer assembly and disassembly.

Treating cells with specific inhibitors (12) of proteins involved in cell wall synthesis did not affect the periodic FtsZ fluctuations (Fig. 1G and table S2). Mutants lacking one of the proteins that regulates the Z-ring (SlmA, SulA, MinC, ClpX, and ClpP) or stabilizes it (ZapA, ZapB, ZapC, ZapD, and MatP) (12) also displayed wild-type Z-ring behavior (Fig. 1, $\mathrm{H}$ and I; fig. S7; and table S3). Thus, Z-ring dynamics are likely due to FtsZ's intrinsic polymerization properties, which are related to its GTPase activity.

To examine whether GTPase activity influences the periodic assembly and disassembly dynamics, we constructed strains each with a single point mutation at the chromosomal fts $Z$ locus (12). The catalytic GTP turnover rate constants $\left(k_{\text {cat }}\right)$ of these mutant proteins in vitro $(12,18,19)$ ranged from $14 \%$ to $71 \%$ of the wild-type $k_{\text {cat }}$ value (fig. S8A and table S1) (12). Because of the high cellular concentration of GTP $(\sim 5 \mathrm{mM})(20)$, the in vivo GTPase activity of these mutants should mainly represent their maximal GTP hydrolysis rate (reflected in $k_{\text {cat }}$ ). Z-ring dynamics were significantly reduced in mutants with lower GTPase activity (Fig. 1, J and K, and table S1). In addition, the subunit exchange rate constants $\left(k_{\mathrm{ex}}\right)$ of these mutants, extracted from PSD curves or obtained from fluorescence recovery after photobleaching (FRAP) (fig. S8, C to E), decreased with $k_{\mathrm{cat}}(12)$ in a manner consistent with coupling to GTP hydrolysis. We observed the same trend using GFP-ZapA (fig. S4F). The fluctuation frequency and $k_{\mathrm{ex}}$ of each mutant were highly correlated with $k_{\text {cat }}$ (Fig. 1, K and L) and with each other (Fig. 1M). Clearly, the periodic Z-ring dynamics are strongly coupled to GTP hydrolysis. 
In some kymographs of cells lacking well-defined midcell Z-rings, zigzags were apparent, indicating directional movement of FtsZ polymers (fig. S9, A and B, and movies S4 to S7). Imaging at higher temporal and spatial resolution (12) revealed that FtsZ polymers exhibited apparently transverse, processive movement across the short axis of the cell, particularly in shorter cells (Fig. 2, A to D; fig. S10; movie S3; and movies S8 to S13). This dynamic movement likely underlies a previous study reporting oscillatory waves of FtsZ (21).

Because individual FtsZ molecules remain stationary in polymers (fig. S11) $(15,22,23)$, the processive movement of FtsZ polymers is most consistent with polymerization at one end and depolymerization at the other, an essential feature of treadmilling. Indeed, treadmilling of FtsZ in vitro has recently been observed (22). Three-dimensional structured illumination microscopy time-lapse imaging directly showed circumferential treadmilling of essentially all Z-rings (Fig. 2, E and F, and movie S14). Using kymographs, we determined the apparent polymerization and depolymerization speeds of each trajectory (Fig. 2C) (12) and their distributions, which were similar (Fig. 2D and table S1), consistent with the classic definition of treadmilling. Hereafter, we refer to the average of these two speeds as the treadmilling speed (table S1).

The treadmilling speed of each mutant strongly correlated with $k_{\text {cat }}$ (Fig. 2G) and with the fluctuation frequency (Fig. 2H). Whole-genome sequencing did not reveal secondary point mutations that could account for these behaviors (table S4). Therefore, although other polymeric properties of FtsZ and/or interactions may also contribute, our data strongly indicate that FtsZ's GTPase activity underlies treadmilling.

Next, we investigated the role of FtsZ treadmilling in cell division. Using scanning electron microscopy, we confirmed that FtsZ mutants with severe disruptions to GTPase activity frequently exhibited slanted, twisted, and/or incomplete septum morphologies (Fig. 3A and fig. S12) (24-26). Although it has been suggested that the pattern of FtsZ localization dictates the shape of the invaginating septum (24), this connection has not been supported by superresolution imaging in several species $(13,15-17,27-31)$, which showed that the wildtype Z-ring is actually composed of heterogeneously distributed, discontinuous FtsZ clusters. Therefore, it is possible that dynamic remodeling of the Z-ring, rather than its localization pattern, is required for symmetrical invagination (32). We hypothesized that the treadmilling dynamics would allow the Z-ring to sample the surface of the growing septum evenly over time, thereby ensuring a uniform spatial distribution of PG synthesis along the septum. To test this hypothesis, we pulse-labeled wild-type cells and three mutants with the fluorescent D-alanine analog HCC-amino-D-alanine (HADA) (33). Blocking the activity of either PBP1b or FtsI, two proteins specific for septal cell wall synthesis, abolished septal HADA incorporation (fig. S13), which suggests that incorporation is specific to new septal PG synthesis. We observed that for labeling pulses shorter than the treadmilling period $(<100 \mathrm{~s}),>60 \%$ and $>80 \%$ of wild-type and $\mathrm{D} 212 \mathrm{G}$ ( Asp $^{212} \rightarrow$ Gly) cells, respectively, displayed punctate incorporation of HADA at septa (Fig. 3, B and C). With longer pulses (>100 s), the percentage of cells with incompletely labeled septa in wild-type cells and in cells of the mild GTPase mutant E250A (Glu ${ }^{250} \rightarrow$ Ala) rapidly dropped (Fig. 3, B and C). However, large fractions of cells of the more drastic mutants D212G and D158A (Asp ${ }^{158} \rightarrow$ Ala) still had incompletely labeled septa even after $1800 \mathrm{~s}$ (Fig. 3, B and C). The total 
integrated septal HADA intensity increased with longer labeling times, but there was no statistically significant difference between the wild type and the three mutants (Fig. 3D and fig. S14). The septum closure rate, which should be proportional to the total septal PG synthesis rate, was essentially the same as that of the wild type for all mutants except D212A and D212G (Fig. 3E and table S5), as found previously (11); septum closure rate measurements in D212A and D212G were unreliable because of the frequent failure of cell division (movie S15). These data are most consistent with FtsZ GTPase activity affecting the spatiotemporal distribution of synthesis, rather than the septal PG synthesis rate.

Next, we reasoned that because FtsZ recruits many proteins involved in septal synthesis, the dynamics of the essential septal transpeptidase FtsI would likely follow that of FtsZ. Using single-molecule tracking in wide-field epifluorescence microscopy, we tracked the movement of a complementing (fig. S15), N-terminal fluorescent protein fusion of FtsI (TagRFP-t-FtsI) at visible constriction sites in FtsZ ${ }^{\mathrm{WT}}$ and three FtsZ ${ }^{\text {mut }}$ backgrounds. Kymographs of TagRFP-t-FtsI fluorescence in FtsZ ${ }^{\mathrm{WT}}$ cells showed diagonal tracks similar to those of FtsZ (Fig. 4A and fig. S16), indicating that individual TagRFP-t-FtsI molecules moved directionally along the septum, in contrast to stationary FtsZ molecules in treadmilling FtsZ polymers (Fig. 2 and fig. S11) (22). The TagRFP-t-FtsI movement was not unidirectional and exhibited large variations in time and in different cells (Fig. 4A and movies S16 to S18). In the FtsZ ${ }^{\text {mut }}$ strains, TagRFP-t-FtsI moved more slowly (Fig. 4, B to D), with mean speed comparable to the corresponding FtsZ treadmilling speed (Fig. 4E). Thus, FtsZ treadmilling guides the directional movement of FtsI and thereby directs the distribution of new septal PG.

Finally, we used ultraperformance liquid chromatography (UPLC) to investigate whether the biochemical composition of the PG itself was altered in D212G cells (12). In both LB and minimal media, D212G cells had shorter glycan strands and greater cross-linking than did wild-type cells (Fig. 4F and fig. S17A), indicating imbalances in the relative levels of septal glycan strand polymerization and cross-linking in Fts ${ }^{\mathrm{D} 212 \mathrm{G}}$ cells. We also observed a large increase in alternative meso-diaminopimelic acid (Dap-Dap) cross-links (Fig. 4F), indicating that the nature of cross-linking reactions was also perturbed. We observed that the differences in PG composition between minicells (round DNA-less cells produced by polar divisions) isolated from FtsZ ${ }^{\mathrm{D} 212 \mathrm{G}}$ and BW25113 $\Delta$ minC cells were similar to the differences seen with intact cells, indicating that the shorter glycans and greater crosslinking are not attributable to aberrant placement of the division site (fig. S17B). A Caulobacter crescentus FtsZ mutant showed opposite changes (less cross-linking and longer glycan strands), along with bulging of the septal wall (34). Thus, the balance between glycan strand polymerization and cross-linking activities is likely an important factor in defining the shape of the septum, with FtsZ coordinating the two enzymatic activities.

Our results show that FtsZ engages in treadmilling powered by GTP hydrolysis to spatially organize the septal PG synthesis machinery (Fig. 4G) without limiting the rate of septum closure. In Bacillus subtilis, FtsZ treadmilling appears to distribute septal PG synthesis and also to dictate the synthesis rate (35). The differences between the two organisms may reflect differential requirements for PG synthesis between Gram-negative and Gram-positive bacteria. Furthermore, whereas the actin homolog MreB relies on wall synthesis for its 
movement, FtsZ exploits its innate treadmilling capacity to control the movement of septal synthesis enzymes. The broad conceptual similarities among FtsZ, MreB, and the movement of cellulose synthase complexes along cortical microtubules in plants (36) suggest that coupling cytoskeletal motion to wall synthesis may be a general strategy across the kingdoms of life to ensure evenly distributed, robust septal wall synthesis and morphogenesis through time-averaging.

\section{Supplementary Material}

Refer to Web version on PubMed Central for supplementary material.

\section{Acknowledgments}

We thank members of the Xiao and Huang labs for helpful discussions and technical assistance; E. Goley, P. Levin, C. Coltharp, J. Buss, G. Lan, J. Lutkenhaus, E. Garner, and G. Squyres for critical discussions; E. Kuru, Y. Brun, and M. VanNieuwenhze for the HADA dye and assistance in its use; J. Lutkenhaus for the MC123 strain; H. Erickson for the FtsZ antibody and internal FtsZ-FP fusion; P. de Boer for the TB43 strain; E. Goley, P. Levin, and their lab members for helpful advice on protein purification and GTPase assay; R. Tsien for the TagRFP-t construct; M. Delannoy for assistance with SEM; K. Esther, D. Jesse, and Nikon for assistance with N-SIM imaging; and J. Franklin for assistance with whole-genome sequencing. Supported by NIH New Innovator award DP2OD006466 and NSF career award MCB-1149328 (K.C.H.); an NSF graduate research fellowship and an ARCS Scholar Awards fellowship (A.M.); NIH grant R01 GM086447, NSF EAGER award MCB- 1019000, and a Hamilton Smith Innovative Research award (J.X.); and NSF grant PHYS-1066293. We are grateful for the hospitality of the Aspen Center for Physics. HADAs were received under a material transfer agreement from M. VanNieuwenhze at Indiana University.

\section{REFERENCES AND NOTES}

1. Nogales E, Downing KH, Amos LA, Löwe J. Nat Struct Biol. 1998; 5:451-458. [PubMed: 9628483]

2. Vaughan S, Wickstead B, Gull K, Addinall SG. J Mol Evol. 2004; 58:19-29. [PubMed: 14743312]

3. Bi EF, Lutkenhaus J. Nature. 1991; 354:161-164. [PubMed: 1944597]

4. Egan AJ, Vollmer W. Ann NY Acad Sci. 2013; 1277:8-28. [PubMed: 23215820]

5. de Boer P, Crossley R, Rothfield L. Nature. 1992; 359:254-256. [PubMed: 1528268]

6. Raychaudhuri D, Park JT. Nature. 1992; 359:251-254. [PubMed: 1528267]

7. Chen Y, Bjornson K, Redick SD, Erickson HP. Biophys J. 2005; 88:505-514. [PubMed: 15475583]

8. Stricker J, Maddox P, Salmon ED, Erickson HP. Proc Natl Acad Sci USA. 2002; 99:3171-3175. [PubMed: 11854462]

9. Erickson HP. Proc Natl Acad Sci USA. 2009; 106:9238-9243. [PubMed: 19478069]

10. Bi E, Lutkenhaus J. J Bacteriol. 1990; 172:5602-5609. [PubMed: 2145263]

11. Coltharp C, Buss J, Plumer TM, Xiao J. Proc Natl Acad Sci USA. 2016; 113:E1044-E1053. [PubMed: 26831086]

12. See supplementary materials.

13. Buss J, et al. PLOS Genet. 2015; 11:e1005128. [PubMed: 25848771]

14. Moore DA, Whatley ZN, Joshi CP, Osawa M, Erickson HP. J Bacteriol. 2016; 199:e00553-16. [PubMed: 27795325]

15. Buss J, et al. Mol Microbiol. 2013; 89:1099-1120. [PubMed: 23859153]

16. Rowlett VW, Margolin W. Biophys J. 2014; 107:L17-L20. [PubMed: 25418183]

17. Strauss MP, et al. PLOS Biol. 2012; 10:e1001389. [PubMed: 22984350]

18. Arjes HA, Lai B, Emelue E, Steinbach A, Levin PA. BMC Microbiol. 2015; 15:209. [PubMed: 26463348]

19. Lu C, Stricker J, Erickson HP. BMC Microbiol. 2001; 1:7. [PubMed: 11394965]

20. Bennett BD, et al. Nat Chem Biol. 2009; 5:593-599. [PubMed: 19561621] 
21. Thanedar S, Margolin W. Curr Biol. 2004; 14:1167-1173. [PubMed: 15242613]

22. Loose M, Mitchison TJ. Nat Cell Biol. 2014; 16:38-46. [PubMed: 24316672]

23. Niu L, Yu J. Biophys J. 2008; 95:2009-2016. [PubMed: 18390602]

24. Addinall SG, Lutkenhaus J. Mol Microbiol. 1996; 22:231-237. [PubMed: 8930908]

25. Bi E, Lutkenhaus J. J Bacteriol. 1992; 174:5414-5423. [PubMed: 1644768]

26. Stricker J, Erickson HP. J Bacteriol. 2003; 185:4796-4805. [PubMed: 12896999]

27. Fu G, et al. PLOS ONE. 2010; 5:e12680. [PubMed: 20856929]

28. Holden SJ, et al. Proc Natl Acad Sci USA. 2014; 111:4566-4571. [PubMed: 24616530]

29. Jacq M, et al. MBio. 2015; 6:e01108-15. [PubMed: 26286692]

30. Lyu Z, Coltharp C, Yang X, Xiao J. Biopolymers. 2016; 105:725-734. [PubMed: 27310678]

31. Li Z, Trimble MJ, Brun YV, Jensen GJ. EMBO J. 2007; 26:4694-4708. [PubMed: 17948052]

32. Lutkenhaus J, Pichoff S, Du S. Cytoskeleton. 2012; 69:778-790. [PubMed: 22888013]

33. Kuru E, et al. Angew Chem Int Ed. 2012; 51:12519-12523.

34. Sundararajan K, et al. Nat Commun. 2015; 6:7281. [PubMed: 26099469]

35. Bisson-Filho AW, et al. Science. 2017; 355:739-743. [PubMed: 28209898]

36. Paredez AR, Somerville CR, Ehrhardt DW. Science. 2006; 312:1491-1495. [PubMed: 16627697] 
A
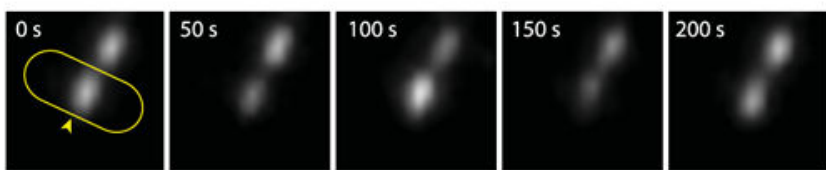

B
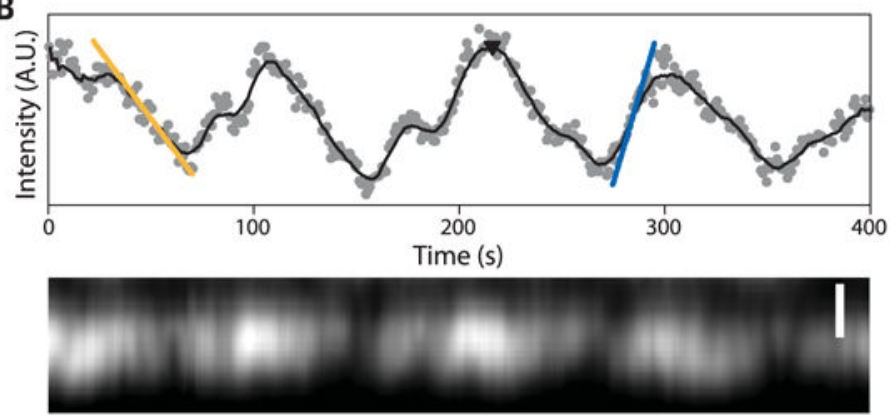

G

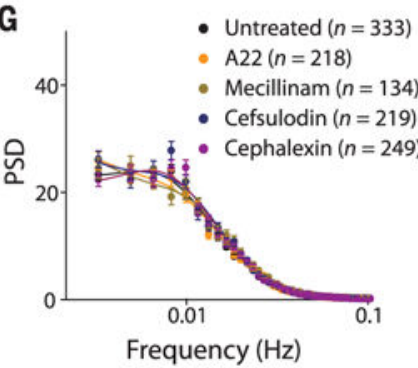

K

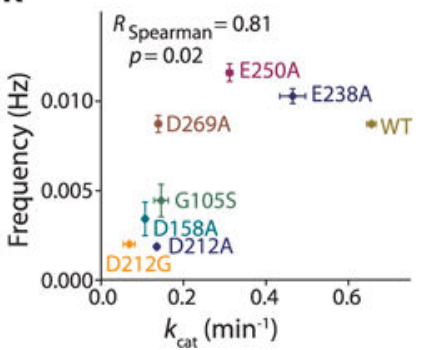

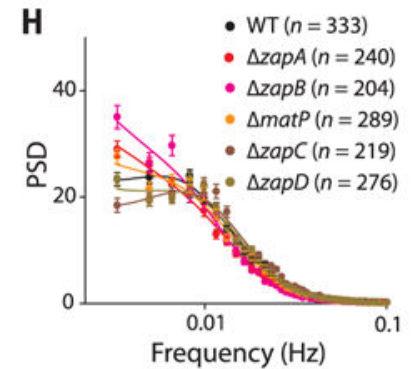

$\mathbf{L}$

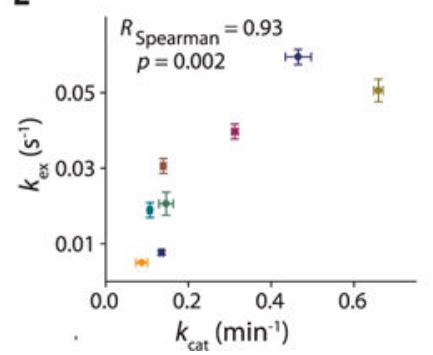

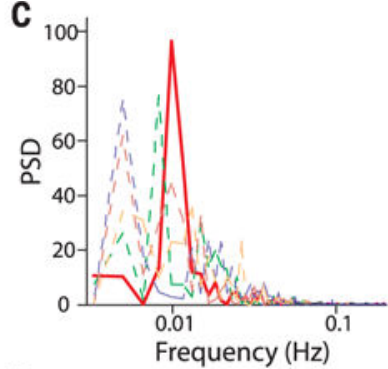

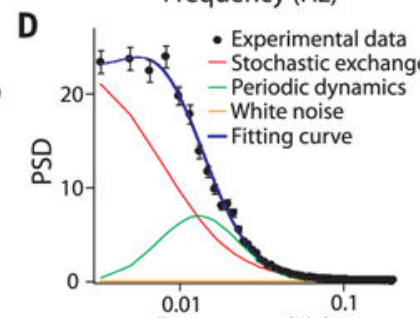

Frequency $(\mathrm{Hz})$

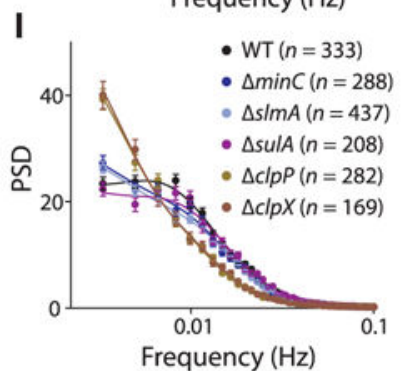

M

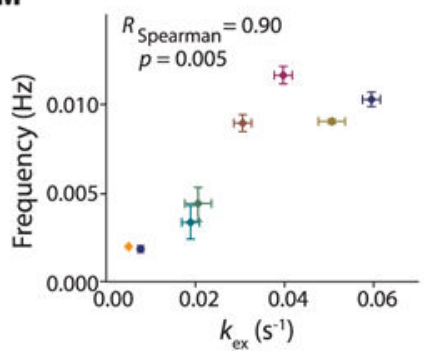

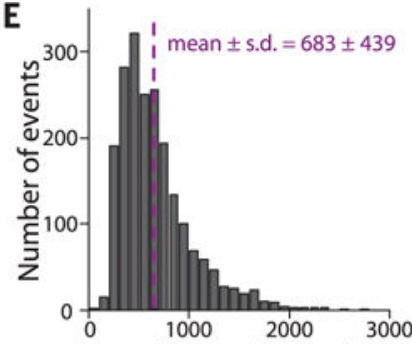

$F$
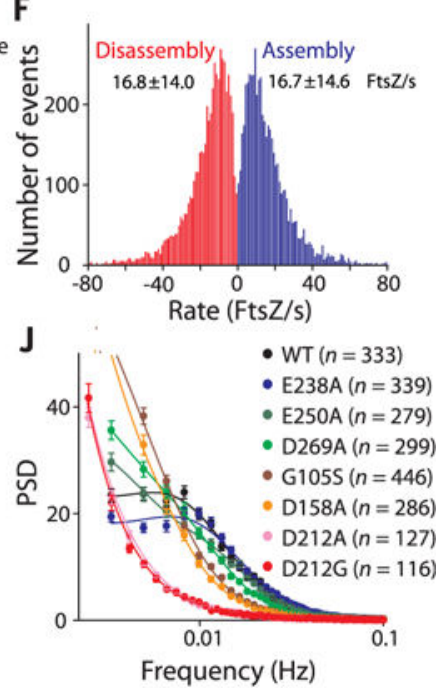

Fig. 1. FtsZ exhibits periodic dynamics coupled to GTPase activity

(A) Montages of live E. coli cells showing periodic FtsZ-GFP (arrowhead) intensity fluctuations. (B) Integrated fluorescence time trace and kymograph of the cell outlined in (A) (1 frame/s; movie S1). The black curve is the moving average (every 20 points) of the raw intensity (gray dots).The assembly and disassembly rates were determined as the maximal slopes along each rise (blue line) and decay (yellow line), respectively. Assembly size was estimated from intensity peaks (black arrowhead) (12). Scale bars, $0.5 \mu \mathrm{m}$. (C) Representative power spectral density (PSD) curves for individual cells (dashed lines), with the one from (B) highlighted in solid red. (D) The mean PSD over all cells ( \pm SEM; $n=333$ cells), fitted with a model (blue curve) that takes into account stochastic subunit exchange between the Z-ring and the cytoplasmic pool (red curve) and the periodic fluctuations (green curve) (12). (E) FtsZ assembly size distribution with $683 \pm 439$ FtsZ and FtsZ-GFP molecules ( $n=2039$ fluorescence peaks). (F) Distributions of assembly and disassembly rates. (G to J) Average PSD curves in drug-treated cells $(\mathrm{G})$, in cells lacking Z-ring stabilizers $(\mathrm{H})$ or regulators (I), and in cells expressing FtsZ GTPase mutants (J). In (G), 
cells were treated with A22 (inhibitor of MreB), mecillinam (inhibitor of the elongationspecific transpeptidase PBP2), cefsulodin (inhibitor of division-specific glycosyltransferase and transpeptidase PBP1b), and cephalexin (inhibitor of the division-specific transpeptidase FtsI) at concentrations above their minimum inhibitory concentration. Error bars denote SEM. (K) The GTPase catalytic turnover rate $k_{\text {cat }}$ is correlated with Z-ring periodic frequency. E238A, Glu ${ }^{238} \rightarrow$ Ala; D269A, Asp $269 \rightarrow$ Ala; G105S, Gly $105 \rightarrow$ Ser. (L) $k_{\text {cat }}$ is correlated with the stochastic exchange rate $k_{\mathrm{ex}}$. $(\mathbf{M}) k_{\mathrm{ex}}$ is correlated with Z-ring periodic frequency. Error bars denote SD. 
A

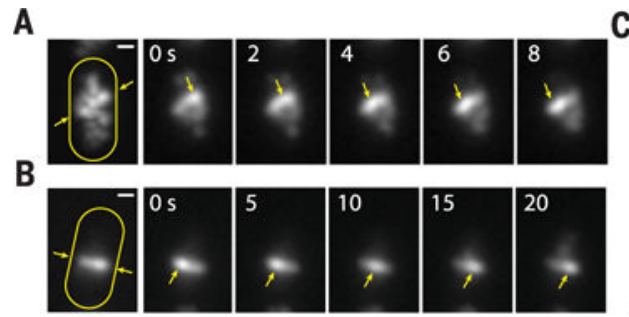

E

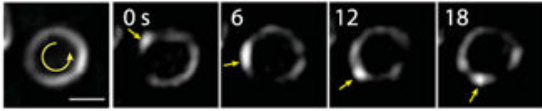

G

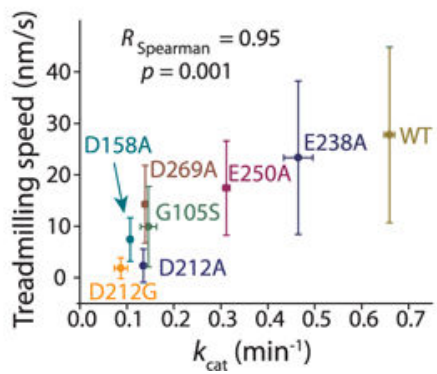

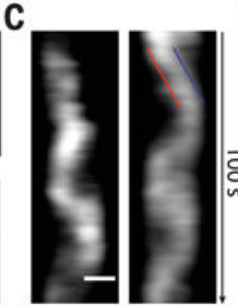

D

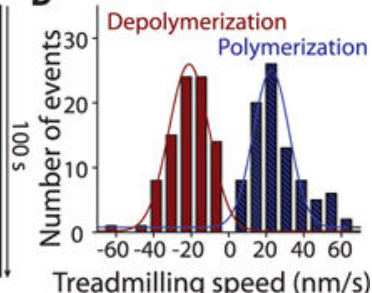

$\mathbf{F}$ $2.3 \mu \mathrm{m}, 360^{\circ}$

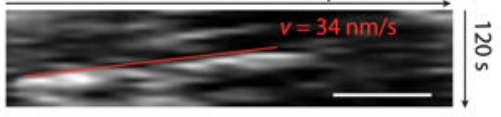

H

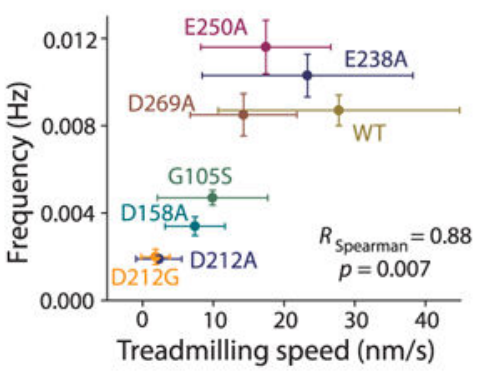

Fig. 2. FtsZ polymers exhibit treadmilling dynamics in live $E$. coli cells

(A and B) Maximum intensity projection (left panels) and montages from time-lapse imaging (movies S8 and S9) of a cell in which a midcell Z-ring was not assembled (A) and a cell with a clearly visible midcell Z-ring (B). (C) Kymographs of the cells in (A) and (B) computed from the intensity along the line between the two yellow arrows. (D) Distributions of polymerization and depolymerization speeds as measured from the leading and trailing edges of individual cells' kymographs [blue and red lines in (C)]. (E) Structured illumination microscopy maximum-intensity projection (left panel) and montage from timelapse imaging of counterclockwise Z-ring treadmilling. (F) Kymograph of fluorescence along circumference of cell in (E). ( $\mathbf{G}$ and $\mathbf{H})$ Treadmilling speeds correlated with $k_{\text {cat }}(\mathrm{G})$ and Z-ring dynamics $(\mathrm{H})$. Error bars denote SD. Scale bars, $0.5 \mu \mathrm{m}$. 
A

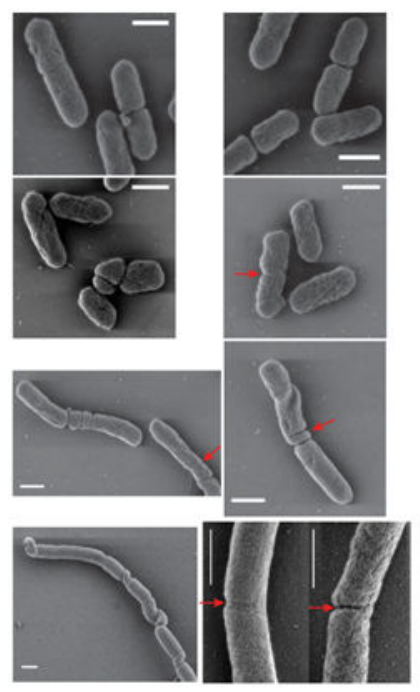

C

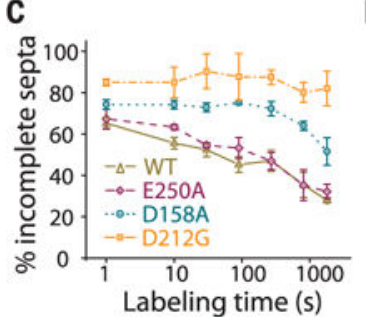

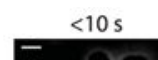

WT

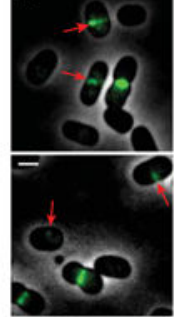

D158A
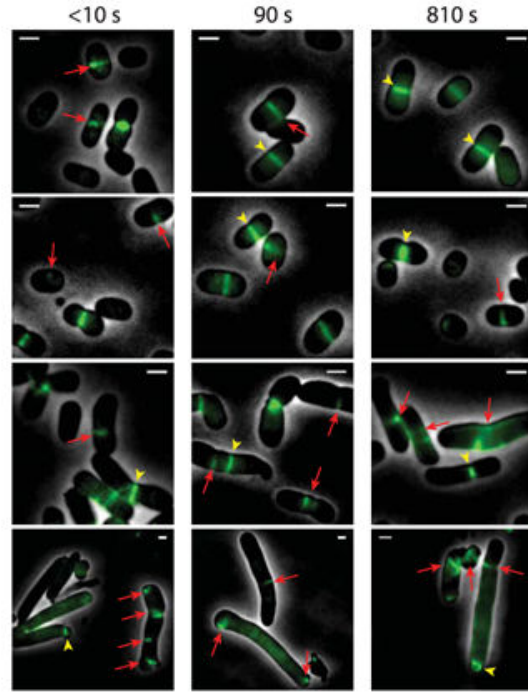

D212G

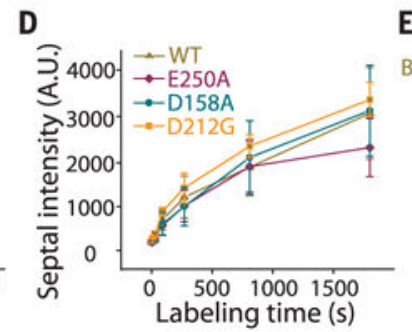

E

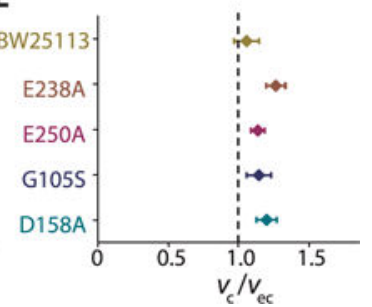

Fig. 3. FtsZ GTPase mutants change the spatial distribution pattern but not the rate of septal PG synthesis

(A) Representative scanning electron microscopy images of FtsZ wild-type, E250A, D158A, and D212G cells. Red arrows denote deformed, asymmetric septa. (B) Representative images of HADA-labeled septa for short ( $<10 \mathrm{~s})$, intermediate (90 s), and long (810 s) labeling pulses. Red arrows and yellow arrowheads denote incomplete and complete septa, respectively. (C) Severe GTPase mutants had large percentages of cells with incompletely labeled septa even for long pulses. (D) Integrated septal HADA fluorescence increased similarly with labeling pulse duration in all strains. (E) The ratio of septum closure rate $\left(V_{\mathrm{c}}\right)$ to elongation rate during constriction $\left(V_{\mathrm{ec}}\right)$ was similar among tested GTPase mutants. Error bars denote SEM. Scale bars, $1 \mu \mathrm{m}$. 

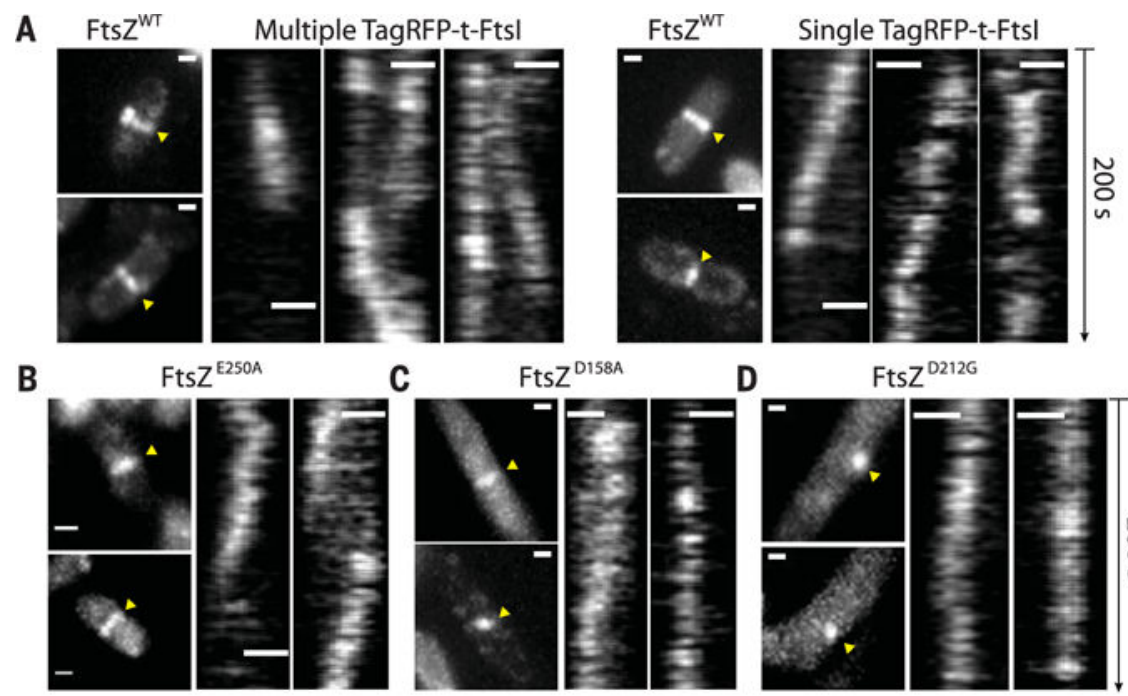

D $\quad \mathrm{FtsZ}^{\mathrm{D} 2126}$

$\mathbf{E}$
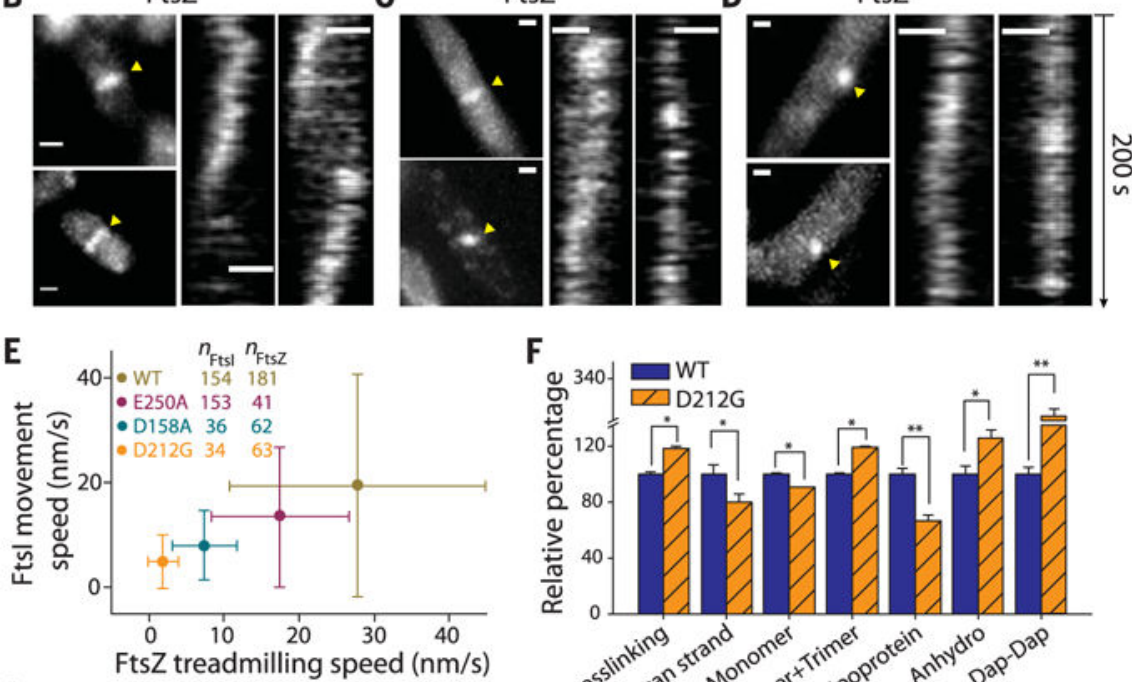

$\mathbf{F}$

G
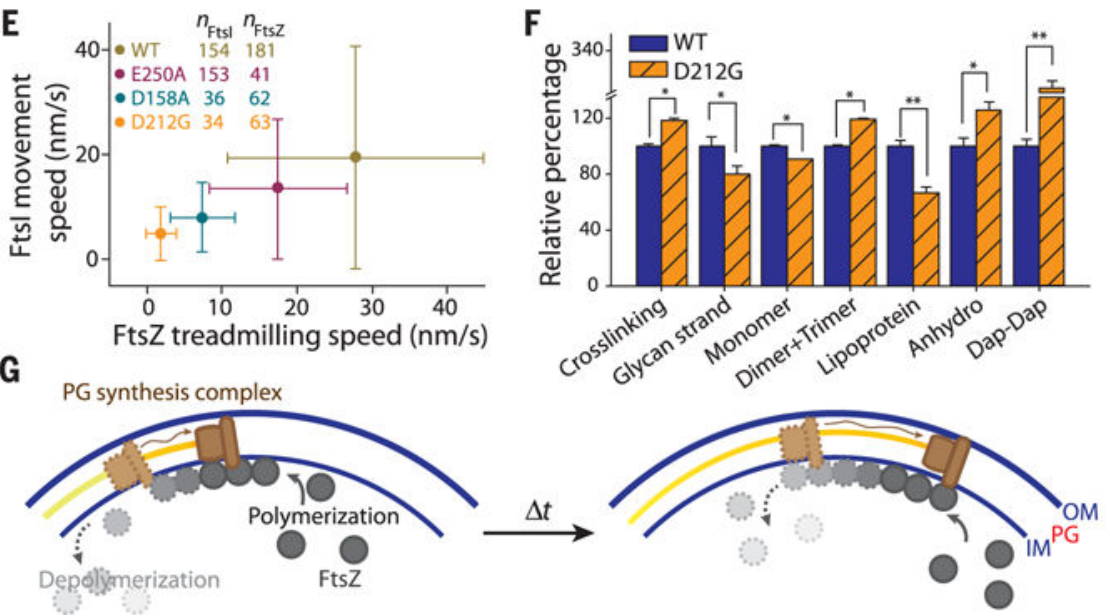

Fig. 4. Altered directional movement of FtsI and septal PG composition in FtsZ ${ }^{\text {mut }}$ cells (A) Directional movement of multiple (left) or single (right) TagRFP-t-FtsI molecules along the septum in FtsZcells. Images with yellow arrowheads are maximum intensity projections; kymograph images are from positions denoted by the arrowheads. (B to D) Examples of TagRFP-t-FtsI directional movement in E250A (B), D158A (C), and D212G (D) cells. (E) Mean TagRFP-t-FtsI movement speed is highly correlated with FtsZ treadmilling speed; error bars denote SD (see table S1). (F) UPLC analysis reveals altered PG composition in Fts ${ }^{\mathrm{D} 212 \mathrm{G}}$ cells. The relative percentage of each component of $\mathrm{D} 212 \mathrm{G}$ was normalized to that of wild-type cells. Error bars denote SD, $n=3$; $* P<0.05$, ** $P<0.01$ (unpaired $t$ test). (G) A schematic model depicting FtsZ treadmilling (gray circles) that drives directional movement (wavy arrows) of the septal PG synthesis machinery (brown rectangles), leading to processive synthesis of new septal PG (yellow). IM and OM, inner and outer membranes; scale bars, $0.5 \mu \mathrm{m}$. 